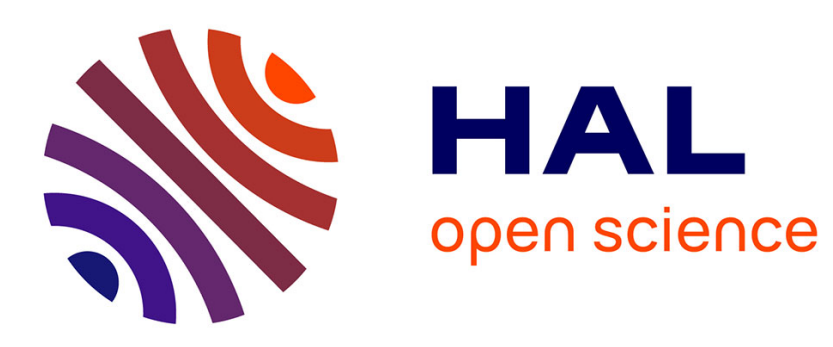

\title{
A mix balanced-modal truncations for power systems model reduction
}

\author{
Mohamed Belhocine, Bogdan Marinescu
}

\section{To cite this version:}

Mohamed Belhocine, Bogdan Marinescu. A mix balanced-modal truncations for power systems model reduction. 13th European Control Conference, Jun 2014, Strasbourg, France. pp.2721 - 2726 , 10.1109/ECC.2014.6862475 . hal-01421794

\section{HAL Id: hal-01421794 \\ https://hal.science/hal-01421794}

Submitted on 30 Jan 2017

HAL is a multi-disciplinary open access archive for the deposit and dissemination of scientific research documents, whether they are published or not. The documents may come from teaching and research institutions in France or abroad, or from public or private research centers.
L'archive ouverte pluridisciplinaire $\mathbf{H A L}$, est destinée au dépôt et à la diffusion de documents scientifiques de niveau recherche, publiés ou non, émanant des établissements d'enseignement et de recherche français ou étrangers, des laboratoires publics ou privés. 


\section{A mix balanced-modal truncations for power systems model reduction}

\author{
M. Belhocine \\ RTE R\&D Division, 9, rue de la porte de Buc \\ 78000 Versailles and SATIE-ENS Cachan \\ 61, Avenue du Président Wilson, 94230 Cachan, France \\ Email: mohamed.belhocine@ens-cachan.fr
}

\author{
B. Marinescu, Member IEEE \\ RTE R\&D Division, 9, rue de la porte de Buc \\ 78000 Versailles and SATIE-ENS Cachan \\ 61, Avenue du Président Wilson, 94230 Cachan, France \\ Email: bogdan.marinescu@rte-france.com
}

\begin{abstract}
In this paper, a new model reduction technique mixing the balanced and modal truncations is proposed for power systems. Usually, only the power transmission lines are approximated such that, when used with the rest of the power system, the phenomena of interest are reproduced. Our approach is structural since it is based on the reduction techniques of the dynamical systems and consider the full power system rather than the lines alone. It is shown that, to obtain a good approximation with the balanced or modal truncations, a large number of dynamics are needed to be kept in the reduced model. By mixing the balanced and modal truncations, a much lower order reduced model is obtained and the behaviour of the full system is faithfully reproduced. This result is useful in practice since it opens the way to improve the numerical simulations and behaviour analysis of power systems by making easy the digital implementations. This new technique is checked by simulation tests in Matlab and EMTP.
\end{abstract}

\section{INTRODUCTION}

A power transmission system is a set of different electric components interconnected by electric transmission lines. Indeed, the full dynamic model of such a system is usually constructed by putting together the dynamic models of each system component with those of transmission lines. Generally, the electric components like machines and loads are finite dimensional systems described by ordinary differential equations (ODE) unlike the transmission lines which are distributed parameters (DP) systems described by partial differential equations (PDE). Therefore, the full model (i.e., all interconnected models) is an infinite dimensional system which can reproduce responses to all kinds of perturbations, particularly electromagnetic transients or wave propagation through the lines.

In practice, this full and detailed model is difficult to be used, and it is usually replaced by one which uses $a$ priori simplified representation of the lines like, for example, the $\pi$-model [7]. However, since the approximation of the line models is a priori imposed and not issued from the analysis of the full model with respect to the purposes of the modelization process (i.e., the phenomena which are supposed to be reproduced with the resulting simplified model), there is no guarantee on the adequacy of the resulting simplified power system model. For this reason, a structural truncation (i.e., based on the analysis of the structure of the whole system) of the full model by using techniques like balanced [6], [1] or modal truncations [3] is a good manner to preserve the phenomena of interest by keeping only the most important dynamics in the reduced model.

The infinite dimensional dynamic models of the transmission power systems have some particularities and the direct application of the balanced or modal truncations give not satisfactory results. This is due to the infinite bandwidth of such systems (resonance peaks infinitely repeated). Physically, this is related directly to the wave propagation phenomena and means that the signals of all frequencies can propagate through the lines. As a consequence, the Hankel singular values involved in balanced truncation decay slowly and there is no significant gap between a previous and next value. This means that all modes are important from an energetic point of view and the balanced truncation give a good result only if a large number of modes are retained in the reduced model. The same conclusion comes out also for modal truncation.

To overcome this difficulty, in [8] it is proposed a balanced truncation but only for a specific, a priori fixed, range of frequency. This is done by weighting the initial dynamic model which represents the physical power system by artificial lowpass blocs which leads to a gap in the Hankel singular values. However, this way of doing is not compliant with our objectives since, as mentioned before, the resulting reduced model must be used for the analysis of dynamic phenomena of which connection to the frequency band restrict is not obvious.

In this paper, we propose an alternative method to obtain much lower order reduced models for power systems by mixing the balanced and modal truncations. More precisely, only some both strongly controllable and observable modes (i.e., more important form the energetic point of view) are kept in the modal truncation.

In addition to the low order of the resulting reduced system, this method is directly related to the structure of the whole system and not only to the one of the transmission lines. As a consequence, the resulting reduced models are better suited to reproduce structural phenomena like interarea and hypo-synchronous oscillations. Also, the adequacy of the truncation to the reduction objectives is quantitatively measured a priori (i.e., before the truncation in terms of magnitude of Hankel singular values), and not a posteriori like for the existing methods for which a simplified model is first proposed by expert intuition for the line model. Next, 
the phenomena of the resulting reduced model of the whole power system are checked a posteriori which leads to an uncertain trial and error process.

\section{POWER SYSTEM MODEL DESCRIPTION}

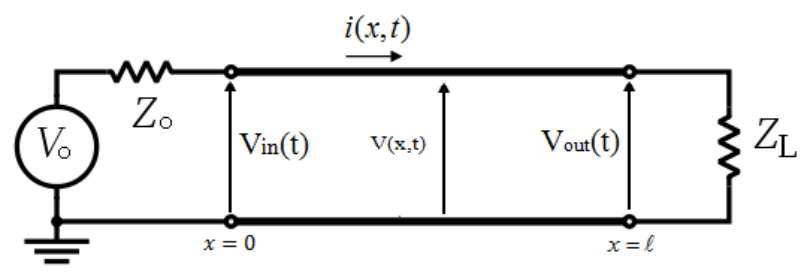

Fig. 1. Power system

The power system in Fig. 1 is composed by three subsystems: A generator assumed to be a voltage source $V_{0}(t)$ behind an impedance $Z_{0}$ (which is the usual simplified model used for voltages studies (see, e.g., [7])), a transmission line of length $\ell$ for which the transverse conductance is neglected, see, e.g., [9] and a resistive load $Z_{L}$.

The dynamic model of the full system is thus an interconnection between the dynamic models of the elements mentioned above.

\section{A. Distributed parameters model for power systems}

First, the distributed parameters model of a transmission line can be written as

$$
\frac{\partial v(x, t)}{\partial x}=-L \frac{\partial i(x, t)}{\partial t}-R i(x, t), \frac{\partial i(x, t)}{\partial x}=-C \frac{\partial v(x, t)}{\partial t}
$$

for $0 \leq x \leq \ell$, where $v(x, t)$ and $i(x, t)$ are respectively the voltage and current along the line, and the positive constant parameters (i.e., independent of frequency) $R, L, C$ are, respectively, the resistance, the inductance and the capacitance per unit length. Next, the interconnection between the transmission line, the load and the generator in Fig. 1 is performed by using the Kirchhoff's laws and the boundary variables of (1) as follows

$$
\begin{aligned}
& \left.v(x, t)\right|_{x=0}=V_{0}(t)-\left.Z_{0} i(x, t)\right|_{x=0} \\
& \left.v(x, t)\right|_{x=\ell}=\left.Z i(x, t)\right|_{x=\ell}
\end{aligned}
$$

Thus, equations (1)-(2) form the full model of the power system (in Fig. 1) which can be written also under the form

$$
\underbrace{\left[\begin{array}{c}
\dot{z}_{1} \\
\dot{z}_{2}
\end{array}\right]}_{\dot{z}(t)}=\underbrace{\left[\begin{array}{cc}
0 & -\frac{1}{C} \frac{\partial}{\partial x} \\
-\frac{1}{L} \frac{\partial}{\partial x} & -\frac{R}{L}
\end{array}\right]}_{\mathcal{A}} \underbrace{\left[\begin{array}{c}
z_{1} \\
z_{2}
\end{array}\right]}_{z(t)}
$$

$$
\mathcal{B} z(t)=V_{0}(t), \quad \mathcal{C} z(t)=y(t), z(0, x)=z_{0}(x),
$$

where $z_{1}(t)=v(x, t), z_{2}(t)=i(x, t)$ and with

$$
\mathcal{B}\left[z_{1}(t) z_{2}(t)\right]^{T}=v(0, t)-Z_{0} i(0, t)=V_{0}(t)
$$

the input and $\mathcal{C}\left[z_{1}(t) z_{2}(t)\right]^{T}=v(\ell, t)=y(t)$, the output.

Equation (3) is a general form of an abstract boundary control system, where $\mathcal{A}, \mathcal{B}, \mathcal{C}$ are operators defined on a given Hilbert space $\mathcal{Z}$ (see, e.g., [4]). It can be written under the infinite dimensional general state representation as

$$
\dot{z}(t)=\tilde{\mathcal{A}} z(t)+\tilde{\mathcal{B}} V_{0}(t), y(t)=\mathcal{C} z(t), z(0, x)=z_{0}(x)
$$

where $\tilde{\mathcal{A}}$ generates an analytic $C_{0}$-semigroup $\mathcal{T}(t)=e^{\tilde{\mathcal{A}} t}$ on the space $\mathcal{Z}$ and the operator $\tilde{\mathcal{B}}$ is deduced from (3) (see, e.g., [4] or [14] for more details and examples).

\section{B. Ad-hoc finite dimensional model of the power system}

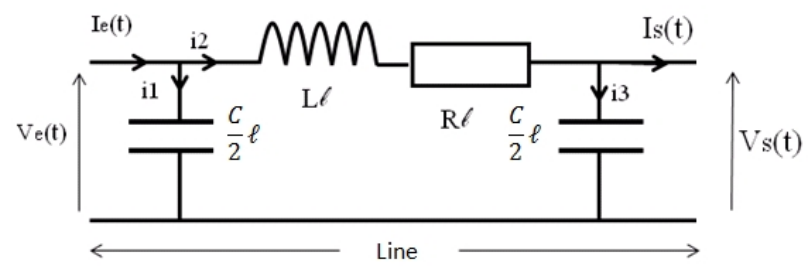

Fig. 2. the $\pi$-model of a transmission line

As previously mentioned, in practice, before aggregating the model (3) of the full power system, the transmission line model (1) is replaced by a finite dimensional equivalent like the $\pi$-model in Fig. 2. Hence, the dynamic model of the full system is given by

$\left[\begin{array}{c}\dot{x}_{1} \\ \dot{x}_{2}\end{array}\right]=\left[\begin{array}{cc}0 & 1 \\ -\left(\frac{2 R \ell+2 Z_{L}}{L C \ell^{2} Z_{L}}\right) & -\left(\frac{2 L+R C \ell Z_{L}}{L C \ell Z_{L}}\right)\end{array}\right]\left[\begin{array}{l}x_{1} \\ x_{2}\end{array}\right]+\left[\begin{array}{c}0 \\ \frac{2}{L C \ell^{2}}\end{array}\right] V_{e}(t)$

with $x_{1}=V_{s}(t)$ and $x_{2}=\dot{x}_{1}$, instead of (3).

Obviously, the model (5) cannot be used instead of (3) to reproduce all physical phenomena of interest in power system analysis. It is generally used only to perform loadflow computations, to analyse transient stability and to design voltage controllers.

\section{STRUCTURAL TRUNCATION OF THE POWER SYSTEM MODEL}

A structural approximation of the power system described by the distributed parameters model (3), is a way to find a finite dimensional reduced model where only the important dynamics (i.e., which have a major impact on the behaviour of the full power system) are kept. In the balanced truncation, e.g., [1] these important dynamics are selected from an energetic point of view based on the Hankel singular values of the input/output system. In the modal truncation, (e.g., [3]) they are selected following their impact on the infinite norm of the input/output system. Both techniques are recalled below and their performances are compared when applied to the simple test system in Fig. 1.

\section{A. Modal truncation}

First, consider the model (4) for the particular case of an open line and ideal voltage source (i.e., the load $Z_{L}=\infty$ and $Z_{0}=0$ in Fig. 1). Obviously, this case does not occur in practice but it is useful to give a first view on the dynamic of the system. As the system becomes simpler in this case, analytic expressions of the transfer function as well as of the trajectories of the system are available. 
More precisely,

$$
\frac{\hat{V}(\ell, s)}{\hat{V}_{0}(s)}=\frac{1}{\prod_{n=1}^{\infty}\left(\frac{4 \ell^{2} L C}{(2 n-1)^{2} \pi^{2}} s^{2}+\frac{\left(\frac{R}{L}\right) 4 \ell^{2} L C}{(2 n-1)^{2} \pi^{2}} s+1\right)}
$$

is the transfer function between the input voltage of sending end $V_{0}(t)$ and the output voltage at distance $\ell$ from the sending end $v(\ell, t)$.

At this stage, a way to obtain a finite dimensional approximation of (4) is the modal truncation of the transfer function (6). For $n=1$, the poles are

$$
p_{1,2}=-\frac{1}{2}\left(\frac{R}{L} \pm \sqrt{\left(\frac{R}{L}\right)^{2}-\frac{\pi^{2}}{8}\left(\frac{8}{\ell^{2} L C}\right)}\right) .
$$

Also, the transfer function of the $\pi$-model (5) for $Z_{L}=\infty$ and $Z_{0}=0$, has the poles

$$
\lambda_{1,2}=-\frac{1}{2}\left(\frac{R}{L} \pm \sqrt{\left(\frac{R}{L}\right)^{2}-\frac{8}{L C \ell^{2}}}\right) .
$$

The only difference between (7) and (8) is factor $\frac{\pi^{2}}{8}$. As the latter factor is close to unity, the first order modal truncation of (6) is close to the $\pi$-model (5) from a structural point of view (see [2] for details). For trajectories, the Fig. 3 shows a comparison of the step responses of the full power system in Fig. 1 for which a $\pi$, respectively, a DP model of line is used. ${ }^{1}$

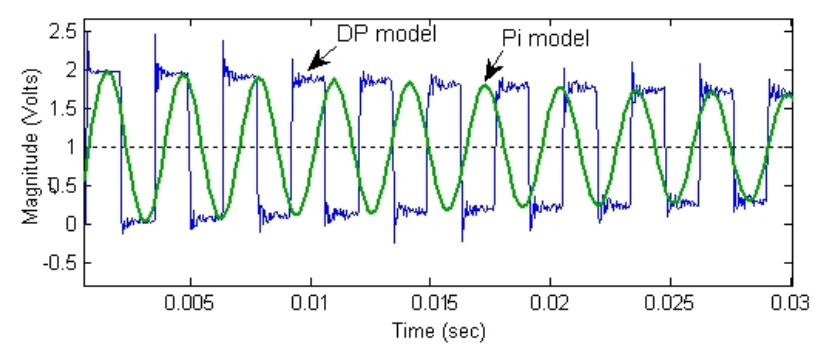

Fig. 3. Step responses with of $\pi$ and DP models for the line

The global shapes of the two trajectories in Fig. 3 are similar. The differences come from the transient dynamics due to the interactions between the waves during the propagation and they are observed in the upper and lower parts of the trajectories provided by the DP model. Thus, the $\pi$ model (first order modal truncation) captures an important component in the behaviour of the DP one but not the dynamics related to the waves propagation. In the latter case, this truncation at the first order is not sufficient and a large number of fractions is needed to be kept in (6).

1) General approach: Starting from a general state representation (4) involving operators, the partial fractions expansion

$$
G(s)=\sum_{k=0}^{\infty} \frac{r_{k}}{s-\beta_{k}}
$$

\footnotetext{
${ }^{1}$ Notice that, to get the trajectories of the DP model, the finite difference method over a finite grid with a step of $10^{-1}$ unit length in $\left[\begin{array}{ll}0 & \ell\end{array}\right]$ was used.
}

of the transfer function can be obtained ( [3], [4], [14]), where $\beta_{k}$ are the poles and $r_{k}$ their residues. The modal truncation consists in keeping only a finite number of these fractions, i.e., the $N^{t h}$ order modal truncation of $G(s)$ is

$$
G^{N}(s)=\sum_{k=0}^{N-1} \frac{r_{k}}{s-\beta_{k}} .
$$

One has the following bound for the $H_{\infty}$ truncation error:

$$
\left\|G(s)-G^{N}(s)\right\|_{\infty} \leq \sum_{k=N}^{\infty} \frac{\left|r_{k}\right|}{\left|\operatorname{Re}\left(\beta_{k}\right)\right|} .
$$

The latter bound shows that the partial fractions in (9) of large $H_{\infty}$ norm are important to be kept in the reduced model in order to lower the truncation error.

2) Numerical approximation: For the general case of the system in Fig. 1 (i.e, with a load) and for power systems of large scale, it is not possible to analytically obtain the partialfraction expansion of the transfer function (9). In this case, these quantities are numerically approximated by finding an approximation $\left(A^{n}, B^{n}, C^{n}\right)$ of the operators $(\tilde{\mathcal{A}}, \tilde{\mathcal{B}}, \mathcal{C})$ in (4) where $n$ is the order of the numerical approximation scheme.

Now, consider the system (4) with the flowing parameters $R=0.5 \Omega / \mathrm{Km}, L=2.10^{-2} \mathrm{H} / \mathrm{Km}, \mathrm{C}=10^{-8} \mathrm{~F} / \mathrm{Km}$, $\ell=50 \mathrm{Km}, Z_{L}=500 \Omega, Z_{0}=0, n=1000$.

Table I presents the poles and the infinite norm of each partial fraction in the transfer function of the $\pi$ and DP model. The infinite norm of the partial fractions of the DP model decreases very slowly and their is no important gap between two consecutive norms of partial fractions (i.e., $\left\|G_{r}\right\|_{\infty} \gg\left\|G_{r+1}\right\|_{\infty}$ ) which might give an index for the modal truncation. For this reason, taking, e.g., $\epsilon=0.1$ as $H_{\infty}$ error bound of (10), i.e, $\sum_{k=N}^{n} \frac{\left|r_{k}\right|}{\left|\operatorname{Re}\left(\beta_{k}\right)\right|}=\epsilon$, the resulting reduced model of the modal truncation is of order 981. As a large number of dynamics are kept in this reduced model, its trajectories are almost the same with the ones provided by

\begin{tabular}{|c|c|c|c|c|}
\hline \multicolumn{3}{|c|}{ full model } & \multicolumn{2}{|c|}{$\pi$-model } \\
\hline $\mathrm{k}$ & poles $\left(\lambda_{k, k+1}\right)$ & $\|\cdot\| \infty$ & poles & $\|\cdot\| \infty$ \\
\hline $1 ; 2$ & $-3,94.10^{2} \pm 6,85.10^{5} i$ & 1,0000789 & $-5,62.10^{2}$ & 1,03 \\
\hline $3 ; 4$ & $-4,14.10^{2} \pm 6,34.10^{5} i$ & 1,0000788 & $-74,621.10^{2}$ & 0,07 \\
\hline $5 ; 6$ & $-4,12.10^{2} \pm 6,38.10^{5} i$ & 1,0000786 & & \\
\hline $7 ; 8$ & $-3,96.10^{2} \pm 6,81.10^{5} i$ & 1,0000782 & & \\
\hline $9 ; 10$ & $-3,97.10^{2} \pm 6,77.10^{5} i$ & 1,0000777 & & \\
\hline $11 ; 12$ & $-4,09.10^{2} \pm 6,46.10^{5} i$ & 1,0000771 & & \\
\hline $13 ; 14$ & $-4,11.10^{2} \pm 6,42.10^{5} i$ & 1,0000764 & & \\
\hline $15 ; 16$ & $-4,06 \cdot 10^{2} \pm 6,53.10^{5} i$ & 1,0000757 & & \\
\hline $17 ; 18$ & $-4,00.10^{2} \pm 6,69.10^{5} i$ & 1,0000745 & & \\
\hline $19 ; 20$ & $-3,99.10^{2} \pm 6,73.10^{5} i$ & 1,0000737 & & \\
\hline 591 & $-5,48.10^{2}$ & 0,975424 & & \\
\hline . & & & & \\
\hline
\end{tabular}
the DP model shown in Fig. 6. This means that this reduced model is adequate to reproduce all phenomena of interest in the power system.

TABLE I

POLES AND $H_{\infty}$ NORM OF EACH PARTIAL FRACTION $\|\cdot\|_{\infty}=\frac{\left|r_{k}\right|}{\left|\operatorname{Re}\left(\lambda_{k}\right)\right|}$ 
It can also be seen from Table I, that only one pole (i.e., $\left.-5.62 .10^{2}\right)$ of the $\pi$-model is close to a pole $\left(-5.48 .10^{2}\right)$ of the DP model. Its rank $(k=591)$ is also very high in the partial fractions expansion of the DP model. Therefore, the reduction link explained above between the $\pi$ and the DP model for open lines, is not true in this case. Thus, from a systemic point of view, when only the transmission lines are replaced by the $\pi$-model of power systems in Fig. 1, the resulting simplified model of the power system cannot be viewed as a structural truncation of the full one. However, the mode of null frequency (i.e., real) kept by the $\pi$-model plays an important role in the behaviour of the DP model as shown in Fig. 6 and it is also kept in the reduced model (of order 981) issued from the modal truncation. In addition, the modes judged important in the DP model according to the $H_{\infty}$ norm are in high frequencies (i.e., pole with large imaginary part) as shown in Table I. Thus the modes of low frequencies (small imaginary parts) are of high rank, and to keep them (like the pole $-5.48 .10^{2}$ ) in the reduced model, a large number of modes should be kept in the end.

It is obvious from the results exhibited in this section that the modal truncation does not give satisfactory results from a practical point of view, since to obtain a good approximation with this method, a large number of modes should be kept which leads to a high order reduced model which cannot be exploited in practice.

\section{B. Balanced truncation}

The balanced truncation is an energy based truncation for which the Hankel singular values are used to keep into the reduced model the highest energy states. As the latter correspond to the most controllable and observable modes, the balanced truncation is performed in two steps. First, get the balanced realization (11)-(13). Next, truncate the system $\mathcal{S}^{b}\left(\tilde{\mathcal{A}}^{b}, \tilde{\mathcal{B}}^{b}, \mathcal{C}^{b}\right)$ at order $r$ by inspecting the Hankel singular values, i.e., $r$ such that $\sigma_{r} \gg \sigma_{r+1}$.

The balanced realisation of the model (4) is given by (see, e.g., [4], [12] or [6])

$$
\begin{gathered}
\tilde{\mathcal{A}}_{i j}^{b}=\frac{\sqrt{\sigma_{i} \sigma_{j}}}{\sigma_{i}^{2}-\sigma_{j}^{2}}\left(\sigma_{j} f_{i}(0)^{T} f_{j}(0)-\sigma_{i} g_{i}(0)^{T} g_{j}(0)\right), i \neq j \\
\tilde{\mathcal{A}}_{i i}^{b}=-\frac{1}{2} f_{i}(0)^{T} f_{i}(0)=-\frac{1}{2} g_{i}(0)^{T} g_{i}(0) \\
\tilde{\mathcal{B}}^{b}=\left[\sqrt{\sigma_{1}} f_{1}(0), \sqrt{\sigma_{2}} f_{2}(0), \ldots\right]^{T} \\
\mathcal{C}^{b}=\left[\sqrt{\sigma_{1}} g_{1}(0), \sqrt{\sigma_{2}} g_{2}(0), \ldots\right]
\end{gathered}
$$

where, $\sigma_{1, \ldots, \infty}$ are the Hankel singular values of the system, $f_{1, \ldots, \infty}(t), g_{1, \ldots, \infty}(t)$, are the singular vectors of the Hankel operator (14).

From (11)-(13) it is clear that $\sigma_{i}, f_{i}, g_{i}$ are firstly needed to balance the system and finally truncate it. In the literature (see, e.g., [4]), these quantities are well defined and their definitions are recalled bellow.
1) Hankel singular values: The Hankel singular values are $\sigma_{i}=\sqrt{\lambda_{i}\left(\mathcal{W}_{o} \mathcal{W}_{c}\right)}$ where $\Gamma$ is the Hankel operator

$$
\Gamma(\bullet)=\int_{0}^{\infty} \mathcal{C} \mathcal{T}(t) \tilde{\mathcal{B}}(\bullet) d t,
$$

2) singular vectors: The singular vectors $f_{i}, g_{i}$ are called Schmidt pair of $\Gamma$ and satisfy

$$
\Gamma f_{i}(t)=\sigma_{i} g_{i}(t), \Gamma^{*} g_{i}(t)=\sigma_{i} f_{i}(t) .
$$

Notice that in the case of infinite dimensional systems $f_{i}(t), g_{i}(t)$ are functions and not vectors as in the finite dimensional case.

Since the $\sigma_{i}$ and the Schmidt pair $\left(f_{i}, g_{i}\right)$ can be computed in practice only for a few cases [10], the algorithms proposed e.g., in [12] and [11] give a numerical approximation of these quantities as in Section III-A.2 and the $r^{t h}$ order truncation is performed when $\sigma_{r} \gg \sigma_{r+1}$.

Notice also that other methods like [13] can be used for finite dimensional approximation.

Consider the system (4) with the same parameters as in Section III-A. Fig. 4 shows the Hankel singular values of (4) obtained by finite dimensional approximation as in Section III-A.2 with $n=1000$. It is clear form this figure that the Hankel singular values decay slowly and there is no significant gap indicating the order of truncation $r$ (i.e., like mentioned above) but just two small gaps (at state 428 the gap is of $2,9.10^{-3}$ between the previous and next state and of $1,2.10^{-3}$ at state 868 ) which means, that from an energetic point of view, all modes play an important role in the inputoutput transfer or equivalently all modes are both strongly controllable and observable which means that the system it is hard to truncate.

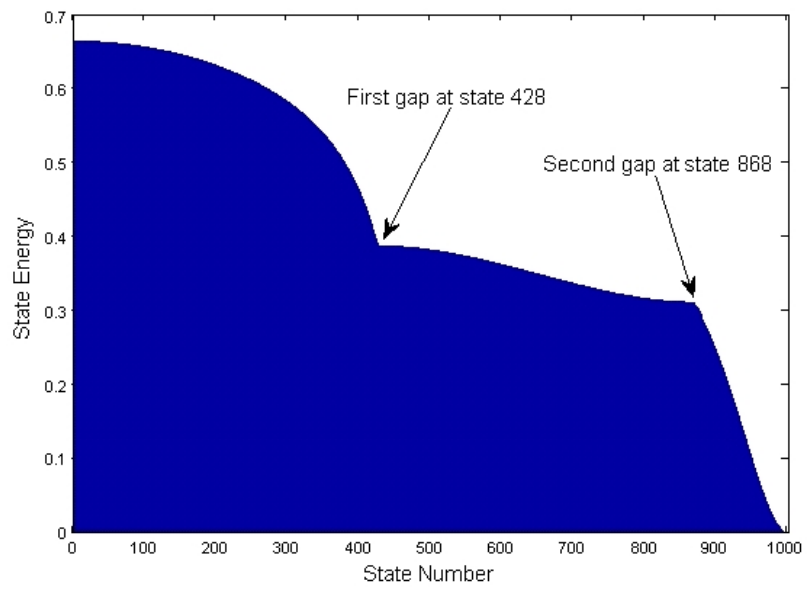

Fig. 4. Hankel singular values of the DP model.

The result of Fig. 4 can be explained also from the gain Bode diagram in Fig. 5. Indeed, the system has an infinite bandwidth and the resonance peaks caused by the infinite number of complex conjugate poles have approximatively the same magnitude and they are infinitely repeated. As a consequence, the system is sensitive to input in any frequency range, i.e., any mode when excited (by putting 
the frequency of input exactly the same with the frequency of the mode) produces the same input-output gain, which means that all modes are important to ensure the bandwidth of the system.

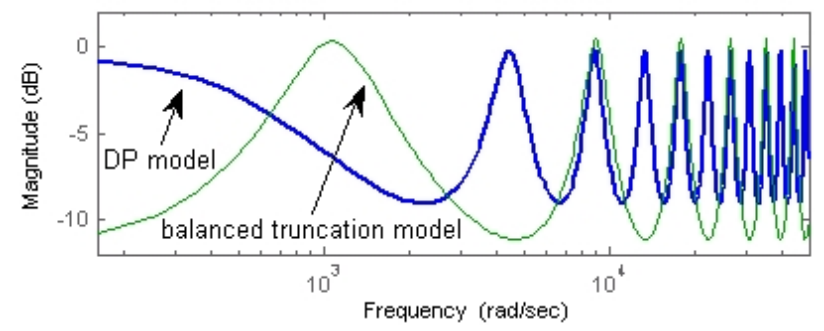

Fig. 5. Bode diagrams of balanced truncation reduced model and DP model.

Indeed, the response of the reduced model computed with $r=428$ which corresponds to the first gap in Fig. 4, are given in Figs. 5 and 6. The frequency response in Fig. 5 shows a bad approximation of the modes of the full model especially for the low frequencies. The step responses presented in Fig. 6 show that the reduced model approximates the full one just at the start and thereafter the trajectories become very different from the ones provided by the DP model and the model implemented in EMTP (Electromagnetic Transient Program) simulator [5].

When $r=868$ which corresponds to the second gap in Fig. 4, the resulting reduced model is much better but contain a too large number of modes. Finally, as for the

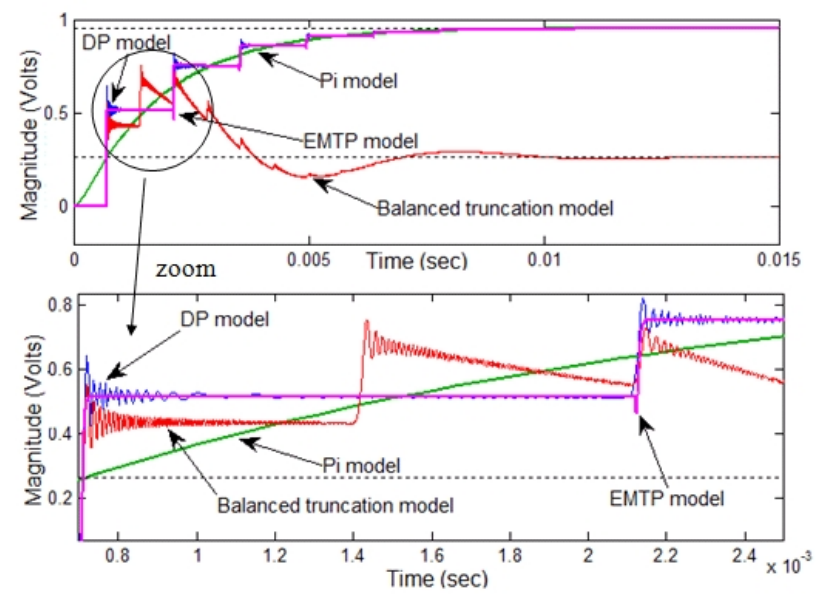

Fig. 6. Step responses comparisons between balanced truncation reduced model, $\pi$, DP and EMTP models.

modal truncation, the number of dynamics to be kept in the reduced model is large and to overcome this difficulty, a new alternative technique is proposed in the next section.

\section{NEW TRUnCATION METHOdOLOGY: MIX BALANCED AND MODAL TRUNCATION}

As none of the balanced or modal truncations can give a low order reduced model for the power system, the idea is to take the advantages of each technique by mixing them.
Indeed, the main advantage of the modal truncation is that the modes are exactly kept but their choice is not always adapted to capture the main dynamics in the system. For the balanced truncation the choice of the dynamics to be kept is useful since it is based on the energy criteria which are close to the physic but the truncation when there is not a significant gap between consecutive Hankel singular values leads to a bad approximation of the modes. The new proposed methodology is a modal truncation for which the modes are chosen from an energetic point of view, i.e., only the modes which participate actively in the states which correspond to large Hankel singular values are kept.

A key point of this approach is the link between the states and the modes of the system which is established using the participation factors [15]. More precisely, let $\left(A^{n}, B^{n}, C^{n}\right)$ be a numerical approximation of operators $(\tilde{\mathcal{A}}, \tilde{\mathcal{B}}, \mathcal{C})$ like in Section III-A.2. The participation factors are the elements of the matrix $P=\left[p_{1}, p_{2}, \ldots, p_{n}\right]$ with $p_{i}=\left[\begin{array}{llll}\phi_{1 i} \psi_{i 1} & \phi_{2 i} \psi_{i 2} & \cdots & \phi_{n i} \psi_{i n}\end{array}\right]^{T}$ where $\phi_{k i}$ is the $k$ th entry of the $i$ th right eigenvector $\Phi_{i}$ of $A^{n}$ and $\psi_{i k}$ is the $k$ th entry of the $i$ th left eigenvector $\Psi_{i}$ of $A^{n}$.

The following properties hold $\Psi_{i} \Phi_{i}=1$ and $\sum_{i=1}^{n} p_{k i}=$ $\sum_{k=1}^{n} p_{i k}=1$.

Thus, each row $i$ of the matrix $P$ contains the net participations of all modes in the $i^{\text {th }}$ state.

Here, the participation factors are used to select the modes to be kept in the modal truncation, i.e., from a state for which the Hankel singular value is large, the participation factors are computed to identify the modes which have large participation in this state and finally keep them in the modal truncation.

Hence, the steps of the methodology we propose are:

1) Compute a balanced realisation as mentioned in Section III-B.

2) Select the states for which the Hankel singular values are large.

3) Compute the participation factors of each mode into the states selected at step 2.

4) Select the modes with major participation in the states selected in step 2 by using the participation factors.

5) Construct the reduced model by modal truncation to the modes found at step 4.

As previously, the system (4) is considered with the parameters given in Section III-A. The reduced model obtained by using this new technique is of order 171 , which is much lower than the reduced models obtained by modal (order 981) or balanced (order 868) truncations. Fig. 7 shows a comparison between the step responses of the new reduced order model, the DP model and the $\pi$-model. The new reduced model, reproduces faithfully the behaviour of the full model which means that all dynamics of interest are kept. Also, Table II shows that the most important (from an energetic point of view) modes in this new reduced model have low frequencies unlike to the modes of Table I given by the modal truncation. This can be seen more precisely from the Bode diagrams of Fig. 8 which shows that the 

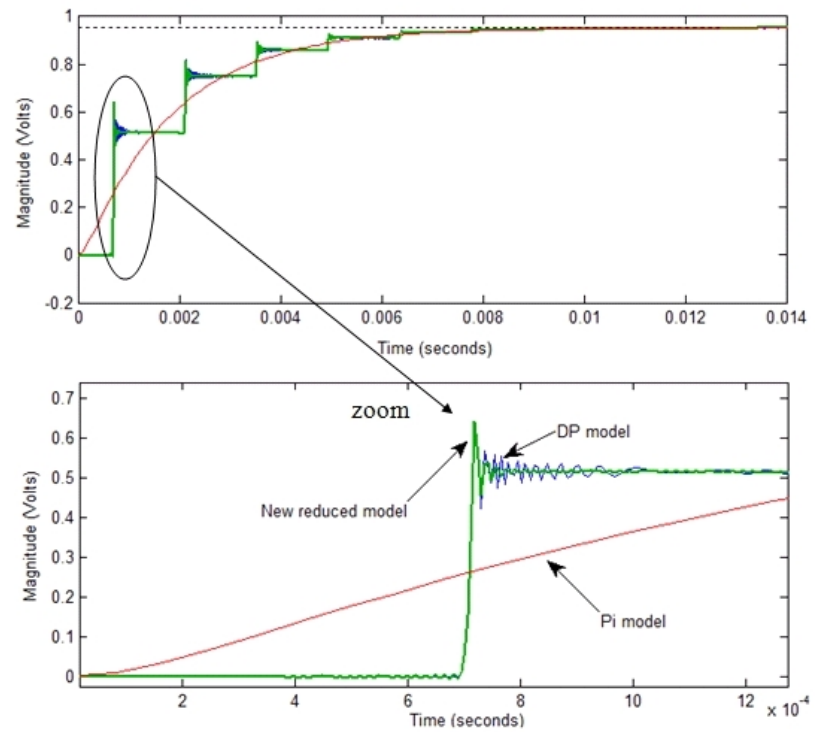

Fig. 7. Comparison between step responses of the new reduced model, $\pi$ and DP models.

TABLE II

FIRST POLES OF THE NEW REDUCED MODEL

\begin{tabular}{|c|c|c|}
\hline states & poles & frequency $(\mathrm{KHz})$ \\
\hline \multirow{2}{*}{$x_{1}$} & $\lambda_{1 ; 2}=5,35.10^{2} \pm 8,88.10^{3} i$ & 1,41 \\
& $\lambda_{3 ; 4}=5.35 .10^{2} \pm 4.44 .10^{3} i$ & 0,70 \\
\hline \multirow{2}{*}{$x_{2}$} & $\lambda_{5 ; 6}=5,35.10^{2} \pm 1,33.10^{3} i$ & 2,11 \\
& $\lambda_{1 ; 2}$ & 1,41 \\
\hline \multirow{2}{*}{$x_{3}$} & $\lambda_{7 ; 8}=5,35.10^{2} \pm 1,77.10^{3} i$ & 2,81 \\
& $\lambda_{5 ; 6}$ & 2,11 \\
\hline
\end{tabular}

new reduced model approximates exactly the DP model in a large frequency bandwidth (up to $3,73.10^{5} \mathrm{rad} / \mathrm{s}$ ). However, the reduced model issued from modal truncation restricted to the order of the new reduced model (172) does not capture the low frequency modes. Consequently, this latter model has a completely inappropriate time behaviour.

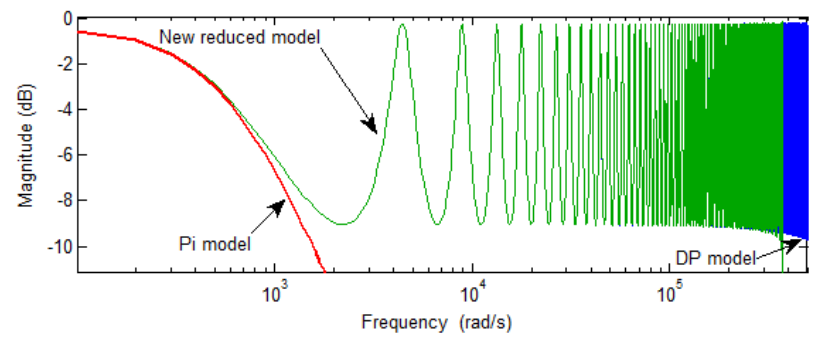

Fig. 8. Bode diagram-comparison between the new reduced, the $\pi$ and DP models.

\section{CONCLUSION}

This paper presents a structural approximation of power systems by a new reduction methodology which mixes the balanced and modal truncations.

From the systemic point of view, this new methodology is advantageous since it allows one to capture the important dynamics of the system with a reduced model of much lower order than the ones provided by modal and balanced based truncations. This advantage is capital in the case where the system has many (infinite number) of important dynamics like the power system treated here.

From the physical point of view, the advantage of this approach is to be structural, i.e., to consider the entire system when the reduction question is addressed and not to substitute a priori simplified models for parts of the system like its transmission lines. The new resulting reduced models are more adequate to reproduce the phenomena of interest in the power system and need less trial and error iterations.

This systemic and modal view will be further exploited to unify and classify some models actually used in different simulators. In this manner, the Transmission Systems Operators (TSOs) could improve their simulation methodology and tools by using a reduced number of simulation models/softwares and better connecting these models to physical phenomena like inter-area and hypo-synchronous oscillations. This will also allow the TSOs to take into account the new electric components (like, e.g., energy sources, High Voltage Direct Current links (HVDC), electronic switches,...).

\section{ACKNOWLEDGMENT}

The authors would like to thank Dr. A. Parisot from RTE and Dr. H. Mounier from LSS for their support.

\section{REFERENCES}

[1] A. C. Antoulas, Approximation of Large-Scale Dynamical Systems. Philadelphia, USA: SIAM, 2005.

[2] M. Belhocine and B. Marinescu, On the connection between used models of power transmission lines. Proc. of the IEEE PES General Meeting Conference, Vancouver, July 21-25 2013.

[3] R. F. Curtain and K. Morris, Transfer functions of distributed parameter systems: A tutorial. Automatica, pp 1101-1116, 2009.

[4] R. F. Curtain and H.J. Zwart, An Introduction to Infinite Dimensional Linear Systems Theory. New York, USA: Springer-Verlag, 1995.

[5] H. W. Dommel, Digital Computer Solution of Electromagnetic Transients in Single and Multiphase Networks. IEEE Trans. Power Apparatus and Systems, vol. PAS-88, No.4, April 1969.

[6] K. Glover, R. F. Curtain, and J. R. Partington, Realisation and Approximation of Linear Infinite-Dimensional Systems with Error Bounds. SlAM J. Control and Optimization, Vol. 26, No. 4, pp 863898, July 1988.

[7] P. Kundur, Power System Stability and Control. New York, USA: McGraw-Hill, pp 202-217, 1994.

[8] S. Lefebvre, Reduced order transmission line models for power system analysis. Electrical Power\& Energy Systems, pp 211-223, 1999.

[9] G. Miano and A. Maffucci, Transmission Lines and Lumped Circuits. USA: Academic Press, 2001.

[10] M. R. Opmeer, Model reduction for distributed parameter systems: A functional analytic view. Proc. of the IEEE American Control Conference (ACC), Montreal:Canada, pp 1418 - 1423, June 27-29 2012.

[11] F. R. Saragih, R. Bambang, and Y. Soeharyadi, Model reduction for infinite dimensional systems using reciprocal transformation. In 7 th Asian Control Conference, pp 1426-1431, 2009.

[12] J. R. Singler, Model Reduction of Linear PDE Systems: A Continuous Time Eigensystem Realization Algorithm. In American Control Conference, Canada, June 27-29 2012.

[13] J. R. Singler, Balanced POD for model reduction of linear PDE systems: convergence theory. Numer. Math, pp 127-164, 2012.

[14] M. Tucsnak and G. Weiss, Observation and Control for Operator Semigroups. Berlin, Germany: Birkhäuser Verlag AG, 2009.

[15] G.C. Verghese, I.J. Péerez-Arriaga and F.C. Schweppe, Selective modal analysis with applications to electric power systems, Part II: The dynamic stability problem. Power Apparatus and Systems, IEEE Transactions on, pp 3126-3134, 1982. 